\title{
Polymer brush bilayers under stationary shear motion at non- linear response regime: An impressive theoretical approach
}

\author{
Mike Edwards \\ Leibniz-Institut für Polymerforchung, Hohe Straße 6, 01069 Dresden, Germany
}

Dated: December 17, 2019

\begin{abstract}
The present article addresses the long-standing problem of the polymer brush bilayers under stationary shear flow at non-linear response regime where the system gets a non-Newtonian fluid. The main idea behind this research would be the fact that the immense lubricity of the polymer brush bilayers originates from a global restructuring that takes place at large shear rates. It is shown here that physical quantities like, stress tensor, viscosity tensor, the friction coefficient and the chain extensions could become dependent on the shear rate at non-Newtonian regime. Apparently, the sub-linear scaling of the physical quantities at large shear rates is solely due to the fact that the chains stretch in the shear direction.
\end{abstract}

Keywords: Polymer brush bilayers, Non-linear response regime, NonNewtonian complex fluids, Stationary shear motion, Density functional theory (DFT), Scaling arguments, Phenomenological arguments 


\section{INTRODUCTION}

Polymers are sort of macromolecular structures which form when a large number of atoms or molecules, i.e. monomers, are connected together ${ }^{1,5}$. The monomers are connected together by sharing their valence electrons and by making a covalent bond. In 1827, R. Brown discovered that the atomic and molecular sized particles undergo thermal fluctuations. The thermal fluctuations of monomers causes a constant change in conformation of the polymer chain. Nevertheless, polymer chains in long term thermal agitation emerge an average length scale representing the size of the chain. Polymers are widely spread in nature with infinitely diverse structures. For instance, linear polymer chains which form when a large number of monomers are connected together in a linear array. DNA strands are a fair example of the linear polymer chains. One of the most functional polymeric structures are polymer brushes $^{2}$. Polymer brushes form when polymer chains are grafted to (from) a substrate with moderately high grafting density. The steric repulsion among monomers of nearby chains stretches the chains in the direction perpendicular to the substrate. Though, the most known brush like structures are formed by linear chains, however, there are brushes formed by nonlinear chains such as polymer stars (linear chains connected in one end) ${ }^{23}$, dendrimers (branched chains connected in one end) etc. Since, the brush covered surfaces have modified surface properties, one could manipulate the surface properties by varying molecular parameters of the brushes. Glycol on the outside of the cell membranes and the aggregans in synovial fluids of mammalian joints are brush like structures that could be found in biological systems ${ }^{2}$. In synovial joints, nature modifies the bones surface to decrease the friction between them by polymer brush bilayers (PBB). It turns out that the sheared PBBs with moderate compression against each other decrease friction up to a several orders of magnitude ${ }^{4,7-9,11-17}$. The reason for such an immense lubricity lays on the fact that the whole system undergoes massive structural changes as the shear rate gets larger than a critical value. In this global restructuring the chains stretch in the shear direction. Apparently, this global restructuring is responsible for reduction of the friction coefficient between the substrates as well as the viscosity of the polymeric solution in the middle. In the present article, the problem of how PBBs respond non-linearly to large stationary shear 
rates is addressed in the context of theoretical Physics. In the next sections, theoretical descriptions are represented followed by the concluding remarks.

\section{THEORETICAL DESCRIPTION}

\section{PBBs at thermal equilibrium}

The PBBs are formed by moderately densely grafting linear chains of degree of polymerization $N$ and the Kuhn length $a$ with grafting density $\sigma$ onto opposing flat plates of distance $D$. Hypothetically, the plates assumed to be located at $z=0$ and $z=D$. The strength of binary correlations between monomers is defined by the second Virial coefficient $b$. As long as the brushes do not interpenetrate, their perpendicular and lateral extensions are calculated as follows ${ }^{3,18,20 ?}$,

$$
\begin{aligned}
& R_{n}=\left(2 a^{2} b \sigma\right)^{1 / 3} N \\
& R_{s}=a N^{1 / 2}
\end{aligned}
$$

Once, they get compressed over each other i.e. $D<2 R_{n}$, the perpendicular and lateral dimensions read as follows ${ }^{20}$,

$$
\begin{aligned}
R_{n} & =\frac{1}{2}\left(\frac{D^{2}}{\chi}+\chi-D\right) \\
R_{s} & =a N^{1 / 2}
\end{aligned}
$$

Where $\chi$ is defines as follows,

$$
\chi=\left(-D^{3}+6 a^{2} b N^{3} \sigma+2 \sqrt{3 a^{2} b N^{3} \sigma\left(3 a^{2} b N^{3} \sigma\right)}\right)^{1 / 3}
$$

It turns out that $R_{n}$ satisfies the following universal power laws,

$$
R_{n} \sim \frac{a^{0.88} b^{0.4} \sigma^{0.38}}{D^{0.1}} N
$$

The above power law indicates that the chains are strongly stretched $\sim N$, however, they weakly stretch as distance $\sim D^{-0.1}$ decreases. The weak stretch upon squeezing the distance 
could be due to interpenetration of the brushes. The equation of state of the PBBs reads as following,

$$
\begin{aligned}
p & =\frac{1}{240 a^{4} b N^{4} D^{4}}\left(435 D^{8}+720 a^{2} b D^{5} N^{3} \sigma-187 D^{7} \chi_{0}^{1 / 3}-192 a^{2} b D N^{3} \sigma \chi_{1} \chi_{0}^{1 / 3}\right. \\
& \left.+187 D^{6} \chi_{0}^{2 / 3}-600 a^{2} b N^{3} \sigma \chi_{1} \chi_{0}^{2 / 3}+2 D^{4} \chi_{0}^{1 / 3} \chi_{2}+4 D^{3} \chi_{0}^{2 / 3} \chi_{3}\right)
\end{aligned}
$$

where the following volumes are introduced for the sake of having shorter formula,

$$
\begin{aligned}
& \chi_{0}=\left(6 a^{2} b N^{3} \sigma-D^{3}+2 \sqrt{3 a^{2} b N^{3} \sigma\left(3 a^{2} b N^{3} \sigma-D^{3}\right)}\right) \\
& \chi_{1}=\left(3 a^{2} b N^{3} \sigma+\sqrt{3 a^{2} b N^{3} \sigma\left(3 a^{2} b N^{3} \sigma-D^{3}\right)}\right) \\
& \chi_{2}=\left(-351 a^{2} b N^{3} \sigma+151 \sqrt{3 a^{2} b N^{3} \sigma\left(3 a^{2} b N^{3} \sigma-D^{3}\right)}\right) \\
& \chi_{3}=\left(-558 a^{2} b N^{3} \sigma+169 \sqrt{3 a^{2} b N^{3} \sigma\left(3 a^{2} b N^{3} \sigma-D^{3}\right)}\right)
\end{aligned}
$$

It turns out that, the equation of state of the PBBs satisfies the following universal power laws,

$$
p \sim a^{-0.88} b^{0.8} \sigma^{1.55}\left(\frac{N}{D}\right)^{2 / 3}
$$

However, at $b \gg 3$, the plot shows the power law $p \sim b^{0.58}$.

\section{PBBs under stationary shear motion at linear response regime}

Once, the properties of the PBBs at equilibrium are known, the transport properties of the PBBs under stationary shear motion could be discussed. In order to find out the transport properties of the PBBs under shear, one needs to know some basics of the fluid dynamics. The dynamics of a Newtonian fluid at small scales is governed by the Stokes equation as follows,

$$
\eta \boldsymbol{\nabla}^{2} \boldsymbol{u}(\boldsymbol{x}, t)=\boldsymbol{\nabla} p
$$

where $\boldsymbol{u}(\boldsymbol{x}, t)$ is the velocity of fluid at position $\boldsymbol{x}$ and time $t$ and $\eta$ is the fluid viscosity. Assuming a shear flow in x-direction, one would need to solve the following equation, 


$$
\eta \frac{\partial^{2} u_{x}}{\partial z^{2}}=\frac{\partial p}{\partial x}
$$

In the absence of external flow, the pressure gradient vanishes i.e. $\frac{\partial p}{\partial x}=0$. The no-slip boundary conditions imply that $u_{x}(z=0)=-v$ and $u_{x}(z=D)=v$, so the solution of Eq. (7) could be read as follows,

$$
u_{x}(z)=\dot{\gamma} z-v
$$

here $\dot{\gamma}=2 v / D$ is the shear rate. It turns out that the fluid velocity behaves linearly in $z$ and it gets vanished at the middle of channel $z=D / 2$. One would get the stress tensor as follows,

$$
\Pi=\eta \nabla \boldsymbol{u}-p \boldsymbol{I}
$$

The normal and shear stress are the only non-zero elements of the stress tensor. The normal stress is the diagonal element and the shear stress is the off-diagonal element of the stress tensor.

$$
\Pi_{n}=p \quad \Pi_{s}=\eta \dot{\gamma}
$$

The above relations show how the normal and shear stresses are related to the equation of state and the viscosity under the stationary shear motions.

At linear response regime, the equation of state of the system does not perturbed by the shear flow. It means that the equation of state of the PBBs at equilibrium holds in the linear response regime as well. The time scale below which the PBBs respond linearly to the shear flow is the largest relaxation time of the chains which is given as follows,

$$
\tau_{c}=\tau_{0} N^{2}=\frac{N^{2} a^{2} \xi}{3 \pi^{2} k_{\mathrm{B}} T}
$$

with $\tau_{0}=\left(a^{2} \xi / 3 \pi^{2} k_{\mathrm{B}} T\right)$ the relaxation time of a monomer. As long as the shear rate is smaller than the critical time scale, the PBBs reside in the linear response regime. It means that the chains do not stretch in the shear direction. The viscosity at linear response regime is an intrinsic property of the system which could be calculated by the equation of state and 
the collision time of the monomers. The collision time of the monomers $\tau$ is an intrinsic time scale representing the average time interval between two subsequent collisions of monomers. Alternatively, $\tau$ is a measure of how long it takes a monomer to travel its mean free path. The collision time for the PBBs is calculated as the mean free path $(D / 2 N \sigma)^{1 / 3}$ divided by the diffusion constant of the monomers $\left(k_{\mathrm{B}} T / N \xi\right)$ which is given as follows,

$$
\tau=\left(\frac{D}{2 \sigma}\right)^{2 / 3} \frac{N^{1 / 3} \xi}{k_{\mathrm{B}} T}
$$

Therefore, the intrinsic viscosity of the PBBs could be calculated and it turns out that the intrinsic viscosity satisfies the following universal power laws,

$$
\eta \sim\left(\frac{\sigma}{a}\right)^{0.88} b^{0.8} N \xi
$$

Having obtained the intrinsic viscosity $\eta$ and the equation of state of the PBBs, one could calculate the shear and normal stresses as well as the friction coefficient. It turns out that the shear and normal stresses and the friction coefficient satisfy the following universal power laws,

$$
\begin{aligned}
\Pi_{s} & \sim\left(\frac{\sigma}{a}\right)^{0.88} b^{0.8} N \xi \dot{\gamma} \\
\Pi_{n} & \sim a^{-0.88} b^{0.8} \sigma^{1.55}\left(\frac{N}{D}\right)^{2 / 3} \\
\mu & =\tau \dot{\gamma} \sim\left(\frac{D}{2 \sigma}\right)^{2 / 3} \frac{N^{1 / 3} \xi}{k_{\mathrm{B}} T} \dot{\gamma}
\end{aligned}
$$

As it is seen, the shear stress and the friction coefficient scale linearly with the shear rate, however, the normal stress and the viscosity are independent from the shear rate. This shows that at the linear response regime, only the shear quantities are affected by the shear flow. The fact that refers back to relaxation of the chains during the shear motion. Another interesting point here is that the shear stress scales linearly with the degree of polymerization $N$, however, the normal stress scales as $\sim N^{2 / 3}$. This will help us in calculation of the shear and normal stresses at the nonlinear response regime. 


\section{PBBs under stationary shear at nonlinear response regime}

As the shear rate increases the chains gradually start to stretch along the shear direction. It turns out that the critical time scale in which the chains start to stretch in the shear direction is equal to the $\tau_{c}$ in Eq. (11). This will throw the system into a different dynamical regime where neither the equation of state nor the linear response (the shear rate exponent) hold. In principle, one could not get any information about the nonlinear response regime where the complex fluid becomes non-Newtonian. The statistical mechanics and the fluid dynamics are unable to approach this problem and find out how the Physical quantities depend upon the molecular parameters ${ }^{22}$. Nevertheless, one could tackle the problem of the non-Newtonian regime and get some basic information by using the phenomenological point of view and the scaling arguments ${ }^{22}$. First of all, the Weissenberg number is introduced as the approperiate scaling variable as follows,

$$
W=\tau_{c} \dot{\gamma}
$$

The Weissenberg numbers lower than one $(W \ll 1)$ correspond to the linear response regime and the Weissenberg numbers $(W \gg 1)$ to the regime of nonlinear response. In this powerful method, the dynamical and transport properties of the PBBs at non-Newtonian regime could be constructed through multiplying the linear response regime quantities by an appropriate universal scaling function $\Xi(W)^{22}$. Let us start by tackling the components of the stress tensor at non-Newtonian regime. The shear stress could be written as follows,

$$
\Pi_{s}(W \gg 1)=\Pi_{s}(W \ll 1) \Xi(W)
$$

The universal scaling function $\Xi(W)$ is in the form of $W^{\alpha}$ with $\alpha$ a real number in $(-\infty,+\infty)$. To find $\alpha$ one could use the phenomenological arguments as follows. The key point is that $\Pi_{s}(W \ll 1) \sim N$ and $\Pi_{n}(W \ll 1) \sim N^{2 / 3}$, and, at the non-Newtonian regime they swap the power laws. It means that, at the non-Newtonian regime, the power laws must be $\Pi_{s}(W \gg 1) \sim N^{2 / 3}$ and $\Pi_{n}(W \gg 1) \sim N$. This comes from the fact that, the scaling of the stress with the degree of polymerization solely is determined by the direction in which the chains are stretched. The golden key in the problem of the PBBs under shear is that 
just the normal and shear stresses swap the power laws of $N$ by passing through the critical shear rate. So, inserting the Weissenberg number as a function of molecular parameters into the Eq. (15) and the fact that $\Pi_{s}(W \gg 1) \sim N^{2 / 3}$, one obtains exponent $\alpha$ by equalizing the power laws of $N$ in both sides of Eq. (15) as follows,

$$
2 \alpha+1=\frac{2}{3} \longrightarrow \alpha=-\frac{1}{6}
$$

After simplifying Eq. (15), one would get the following universal power law for $\Pi_{s}(W \gg$ 1),

$$
\Pi_{s}(W \gg 1) \sim\left(3 \pi^{2} k_{\mathrm{B}} T\right)^{1 / 6} \frac{\sigma^{0.88} b^{0.8} N^{2 / 3} \xi^{5 / 6}}{a^{1.22}} \dot{\gamma}^{5 / 6}
$$

where it has been considered that $\dot{\gamma}^{1+\alpha}$. As it is seen, the shear stress scales sub-linearly with the shear rate. The same phenomenological argument could be used to calculate $\Pi_{n}(W \gg 1)$. Here, one must consider the fact that at $(W \gg 1), \Pi_{n}(W \gg 1) \sim N$ as the chains stretch in the shear direction now. The calculations lead to the following universal power law for the $\Pi_{n}(W \gg 1)$,

$$
\Pi_{n}(W \gg 1) \sim \frac{b^{0.8} \sigma^{1.55} N \xi^{1 / 6}}{\left(3 \pi^{2} k_{\mathrm{B}} T\right)^{1 / 6} a^{0.54} D^{2 / 3}} \dot{\gamma}^{1 / 6}
$$

So, it turns out that $\Pi_{n}(W \gg 1) \sim \dot{\gamma}^{1 / 6}$, which tells us that the normal stress scales sub-linearly with the shear rate as well. Here, the exponent $\alpha$ has been satisfied the equation $2 \alpha+\frac{2}{3}=1$ and it sets to $\alpha=1 / 6$. The kinetic friction coefficient at non-Newtonian regime could be calculated as follows,

$$
\mu(W \gg 1)=\frac{\Pi_{s}(W \gg 1)}{\Pi_{n}(W \gg 1)} \sim 3.09 \frac{\left(k_{\mathrm{B}} T\right)^{1 / 3}(D \xi)^{2 / 3}}{a^{0.68} N^{1 / 3} \sigma^{0.67}} \dot{\gamma}^{2 / 3}
$$

It is interesting to see that the friction coefficient has been ended up with the universal power law $\mu(W \gg 1) \sim \dot{\gamma}^{2 / 3}$ because its sub-linear scaling is confirms the result of the work already publishes ${ }^{4}$.

At the non-Newtonian regime, the shear flow is so strong that decomposes even the viscosity into shear and normal components. One could calculate the shear viscosity as follows, 


$$
\eta_{s}(W \gg 1)=\frac{\Pi_{s}(W \gg 1)}{\dot{\gamma}} \sim\left(3 \pi^{2} k_{\mathrm{B}} T\right)^{1 / 6} \frac{\sigma^{0.88} b^{0.8} N^{2 / 3} \xi^{5 / 6}}{a^{1.22}} \dot{\gamma}^{-1 / 6}
$$

and the normal viscosity could be calculated as,

$$
\eta_{n}(W \gg 1)=\frac{\Pi_{n}(W \gg 1)}{\tau} \sim \frac{2^{2 / 3}\left(k_{\mathrm{B}} T\right)^{5 / 6} \sigma^{2.21} b^{0.8} N^{2 / 3}}{\left(3 \pi^{2}\right)^{1 / 6} a^{0.54} D^{4 / 3} \xi^{5 / 6}} \dot{\gamma}^{1 / 6},
$$

The same argument with a phenomenological approach could be utilized to calculate the lateral and perpendicular extensions of the PBB chains at non-Newtonian regime. One could write the lateral and perpendicular extensions at $(W \gg 1)$ as follows,

$$
\begin{aligned}
& R_{s}(W \gg 1)=R_{s}(W \ll 1) \Xi(W), \\
& R_{n}(W \gg 1)=R_{n}(W \ll 1) \Xi(W),
\end{aligned}
$$

The phenomenological argument that helps calculating the universal scaling function which is in the form $\Xi(W)=W^{\alpha}$ is again related to the swap between normal and perpendicular extensions at $(W \gg 1)$. At $(W \ll 1)$ one would expect $R_{s}(W \ll 1) \sim N^{1 / 2}$ and $R_{n}(W \ll 1) \sim N$. However, at $(W \gg 1)$, one would expect a swap between normal and lateral extensions to become $R_{s}(W \gg 1) \sim N$ and $R_{n}(W \gg 1) \sim N^{1 / 2}$. By using these phenomenological arguments, one could obtain the following universal power laws for the lateral and normal extensions,

$$
\begin{aligned}
R_{s}(W \gg 1) & \sim \frac{a^{3 / 2} N \xi^{1 / 4}}{\left(3 \pi^{2} k_{\mathrm{B}} T\right)^{1 / 4}} \dot{\gamma}^{1 / 4} \\
R_{n}(W \gg 1) & \sim\left(\frac{3 \pi^{2} k_{\mathrm{B}} T}{\xi \dot{\gamma}}\right)^{1 / 4} \frac{N^{1 / 2} a^{0.38} \sigma^{0.38} b^{0.4}}{D^{0.1}}
\end{aligned}
$$

The results above has been obtained as the scaling variable $\alpha$ has been calculated from $2 \alpha+\frac{1}{2}=1$ for the lateral extension and from $2 \alpha+1=1 / 2$ for the normal extension. As one could realize, the lateral extension scales sub-linearly with the shear rate and increases as $\sim \dot{\gamma}^{1 / 4}$, however, the normal extension decreases by the shear rate as $\sim \dot{\gamma}^{-1 / 4}$. 


\section{CONCLUDING REMARKS}

One of the most fascinating areas of Physics that has attracted physicists interests in the last decades has been the soft matter and Biological Physics. The significance of these research area would get more distinguished when they deal with the mechanisms occur in the human or animals body. The PBBs which are present in mammalian joints are one of those significant systems which has attracted an abundant attention. What makes the PBBs high-priority for research is their prodigious ability in reducing friction. In a simple word, when the PBBs are subjected to a low shear rate, they behave very close to the simple fluids and respond linearly with the shear rate. Nevertheless, at sufficiently large shear rates the sub-linear responses loom. For decades, it has been thought that the reduction of the interpenetration between the opposing brushes would be responsible for that sub-linear scaling ${ }^{4}$. The present research that is based on the statistical mechanics, the density functional theory (DFT) and the scaling theory, brings a new idea up that, apparently, a global restructuring could be responsible for the effect. The global restructuring takes place such that the chains stretch in the shear direction rather than in the perpendicular direction. It is suggested that this could reshape the system to a quite distinct conformation. The results presented here, would be a true triumph for the human intellect as it integrates the statistical mechanics, the density functional theory (DFT), the fluid dynamics and scaling theory to approach dynamics of one of the most intricate problems in theoretical Physics of complex fluids, polymer solutions, soft matter and biological systems etc. 


\section{References}

1. Rubinstein, M. and Colby, R.H., Polymer Physics, OUP Oxford (2003)

2. Advincula, R.C. and Brittain, W.J. and Caster, K.C. and Rühe, J., Polymer Brushes, Wiley-VCH, Weinheim (2004)

3. Schwabl, F. and Brewer, W.D., Statistical Mechanics, Advanced Texts in Physics, Springer Berlin Heidelberg, 313-316 (2006)

4. Kreer, T, Polymer-brush lubrication: a review of recent theoretical advances, Soft Matter, 12,3479 (2016)

5. Gennes, Pierre Gilles, Scaling Concepts in Polymer Physics, Cornell University Press, 74-75 (1979)

6. Hirz, S. J., Modeling of Interactions Between Adsorbed Block Copolymers, University of Minnesota, Minneapolis, MN (1988)

7. MILNER, S. T., Polymer Brushes, 251, 4996, 905-914, Science (1991)

8. Halperin, A. and Tirrell, M. and Lodge, T. P., Tethered chains in polymer microstructures, Macromolecules: Synthesis, Order and Advanced Properties, Springer Berlin Heidelberg, Berlin, Heidelberg, 31-71 (1992)

9. Szleifer, I. and Carignano, M. A., Wiley-Blackwell, Tethered Polymer Layers, Advances in Chemical Physics, 165-260 (2007)

10. Milner, S. T. and Witten, T. A. and Cates, M. E., A Parabolic Density Profile for Grafted Polymers, EPL (Europhysics Letters), 5, 5, 413 (1988)

11. Zhulina, E.B. and Borisov, O.V., Structure and stabilizing properties of grafted polymer layers in a polymer medium, Journal of Colloid and Interface Science, 144, 2, 507-520 (1991) 
12. Galuschko, A. and Spirin, L. and Kreer, T. and Johner, A. and Pastorino, C. and Wittmer, J. and Baschnagel, J., Frictional Forces between Strongly Compressed, Nonentangled Polymer Brushes: Molecular Dynamics Simulations and Scaling Theory, Langmuir, 26, 9, 6418-6429 (2010)

13. Kreer, Torsten and Binder, Kurt and Mser, Martin H., Friction between Polymer Brushes in Good Solvent Conditions:? Steady-State Sliding versus Transient Behavior, Langmuir, $19,18,7551-7559(2003)$

14. Kreer, T. and Müser, M. H. and Binder, K. and Klein, J., Frictional Drag Mechanisms between Polymer-Bearing Surfaces, Langmuir, 17, 25, 7804-7813 (2001)

15. Klein, Jacob and Perahia, Dvora and Warburg, Sharon, Forces between polymer-bearing surfaces undergoing shear, Nature, 352, 143 (1991)

16. Taunton, Hillary J. and Toprakcioglu, Chris and Fetters, Lewis J. and Klein, Jacob, Forces between surfaces bearing terminally anchored polymer chains in good solvents, Nature, 332, 712 (1988)

17. Klein, Jacob and Kumacheva, Eugenia and Mahalu, Diana and Perahia, Dvora and Fetters, Lewis J., Reduction of frictional forces between solid surfaces bearing polymer brushes, Nature, 370, 634 (1988)

18. Safran, S., Statistical Thermodynamics Of Surfaces, Interfaces, And Membranes, Addison-Wesley Publishing Company, The advanced book program (1994), p. 229-231

19. Doi, M. and Edwards, S. F., The theory of polymer dynamics, Clarendon Press. Oxford (1986), p. 95

20. Farzin, M., Polymer brush bilayers at thermal equilibrium: A theoretical study, 2018, https://doi.org/10.1101/316141.

21. Edwards, M., Polymer brush bilayers under stationary shear motion at linear response regime: A theoretical approach (2019) http://dx.doi.org/10.1101/565796 
22. These information have been extracted from my handwritten lecture notes at the Prof. J. U. Sommer and Dr. T. Kreer lectures on Theoretical polymer and soft matter Physics at Technical University of Dresden, Germany on winter semester 2013 and summer semester 2015.

23. Edwards, M., Interpenetration between a polymer brush and a polymer star at thermal equilibrium: A theoretical approach, (2018) http://dx.doi.org/10.1101/489559. 